\title{
Encontrar problemas y creatividad *
}

\author{
Jacob M. Getzels \\ Mibaly Csikszentmibalyi
}

Se dice a menudo (Wertheimer, 1945; Getzels y Jackson, 1962) que las explicaciones psicológicas del pensamiento creador han seguido, tradicionalmente, dos enfoques principales. Uno pone énfasis en la racionalidad. Sostiene que el pensamiento supone una aproximación a la verdad lógica. Sea verdad o mentira, es una clase de aserciones y proposiciones. Se pueden hacer inferencias a partir de ellas y aplicar operaciones formales para probar su validez. Las combinaciones hacen posible derivar "nuevas» proposiciones y la lógica establece las distintas formas de silogismos que garantizan el que la solución sea correcta.

La deducción a través de la lógica y la racionalidad es el sustrato de la producción creadora; el descubrimiento del planeta Neptuno, por ejemplo, fue resultado de este proceso. $\mathrm{La}$ inducción - con su confianza en la experiencia y la experimentación- también depende de la lógica y la racionalidad. Aquí, el enfoque no está en deducir relaciones válidas desde proposiciones generales, sino en acumular hechos y establecer sus relaciones hasta que se encuentren nuevas generalizaciones válidas. Tanto si el pensamiento procede por deducción como por inducción, el énfasis está en la racionalidad y la lógica.

Un segundo enfoque es el del asociacionismo clásico. Desde este punto de vista, el pensamiento es una cadena de ideas, o, más exactamente, una cadena de conexiones estímulo-respuesta. No hay diferencias entre los denominados procesos mentales superiores e inferiores, entre ensayo y error, entre pensamiento lógico y original. Las nuevas ideas resultan de asociaciones de ideas viejas por ensayos y errores. El hábito y la experiencia pasada -la recuperación más que la razón- son las características fundamentales del pensamiento. La distinción entre formas superiores $e$ inferiores de pensamiento es más aparente que real; no hay diferencias cualitativas fundamentales, sino diferencias cuantitativas. Como Thorndike señala: «En su naturaleza más profunda, las formas superiores de las operaciones inte-

* Tomado de J. W. Getzels, y M. Csikszentmihalyi: The Creative Vision: A Longitudinal Study of Problem Finding in Art. Nueva York: John Wiley and Sons, 1976. Reproducido con autorización ${ }^{\circ}$ de esta traducción. Estudios de Psicologia, 1984. 
lectuales son idénticas a la mera asociación o formación de conexiones, dependen de la misma clase de condiciones fisiológicas, pero necesitan mayor cantidad de ellas" (Thorndike, 1926, 415; subrayado en el original).

Wertheimer, que representa a los gestaltistas, fue muy crítico con ambas perspectivas. El pensamiento no procede paso a paso, sea por lógica o por asociación. Procede por la reestructuración de configuraciones. El organismo experimenta primero una situación problema en el ambiente $S_{I}$, que actúa como un desencadenante del proceso y luego se alcanza una situación de solución, $S_{2}$, después de la cual termina el proceso. La situación problema es estructuralmente incompleta, mantiene una laguna o un "problema estructural». La «situación-solución» es estructuralmente completa; la laguna se cubre, el problema estructural ha desaparecido.

Cuando la tarea es hallar el área de un rectángulo, el proceso de pensamiento desde $S_{1}$ a $S_{2}$ es claro. Supongamos que se presenta este problema: ¿Cuál es el área de un rectángulo con lado $\mathrm{A}$ y base $\mathrm{B}$ ? $\mathrm{El}$ modo de solución de este problema, como de todos los problemas en los que está implicado el pensamiento, se caracteriza por una «agrupación», «reorganización» y «estructuración» de los elementos dentro de conjuntos y subconjuntos con referencia a la tarea. Las características estructurales y requerimientos de la situación desarrollan ciertos esfuerzos y tensiones en el pensador. Estos esfuerzos y tensiones producen vectores en la dirección de convertir la situación problema $S_{1}$, a través de una línea «estructuralmente consistente", en la situación solución $S_{2}$ (Wertheimer, 1945, 41-42).

Este modelo se adapta bien a las condiciones en las que el problema se puede definir por una solución reconocible. Pero existen condiciones que no se pueden incluir, ciertamente, en la clasificación $\mathrm{S}_{1}-\mathrm{S}_{2}$; los procesos del pensamiento no comienzan con un problema ya formulado. Por el contrario, la cuestión reside precisamente en percatarse de que la situación no es lo buena como debería ser, que necesita ser perfeccionada. En este caso, el primer paso es darse cuenta de que existe un problema: "encontrar» el problema. Este es el caso del científico o el matemático que deben plantear su propio problema, o en palabras de Einstein, "cometer su propio crimen»; o del artista que lleva una vida retirada, creando literalmente un problema que no existe antes. El problema no se ha presentado, debe ser descubierto.

Wertheimer distingue entre situaciones problema presentadas (con un $S_{1}$ dado) y situaciones problema descubiertas (sin él). Escribe acerca de estas últimas lo que él reconoce como característico del trabajo creador:

«El proceso comienza como en otros procesos creadores en arte y música, por la representación de algunas características en un $\mathrm{S}_{2}$ que se va a crear. El artista se ve conducido hacia su cristalización, concreción o realización total... Mientras que lo que sucede en ejemplos del tipo $S_{1} \ldots S_{2}$ está estructuralmente determinado por la naturaleza de $S_{1}$, o de $S_{1}$ en relación a $S_{2}$, aquí está determinado por las características estructurales en la representación $S_{2}$, aunque $S_{2}$ sea todavía incompleta, todavía vaga. Esto, en alguna medida, cambia la naturaleza dinámica del diseño presentado antes (esto es en la situación $S_{1}-S_{2}$ )..» (Wertheimer, 1945, 197 198).

Cuando la situación es del tipo $S_{1}-S_{2}$, la formulación de Wertheimer es fructífera y muchos trabajos empíricos de solución de problemas y pensamiento creador se han hecho en estos términos. Pero cuando la situación es tal que $S_{1}$ no juega ningún papel o apenas lo juega y los individuos deben encontrar su propio problema, el modelo es menos satisfactorio. Falla al tratar dos temas antes mencionados, pero que deja sin respuesta: la fuente del problema real que es encontrado o creado y la fuente del impulso hacia su realización en un producto creativo. 


\section{Estudios}

Las explicaciones psicoanaliticas parecen estar dirigidas a cuestiones de este tipo. Las descripciones del psicoanálisis se focalizan en los orígenes de la creatividad y en las motivaciones para la formulación y solución de los problemas creativos. Aunque Freud trató de la creatividad en numerosos contextos, no dejó ninguna formulación concluyente sobre este tema, $y$, a partir de su trabajo, se han derivado diversas perspectivas. Sin embargo, parece haber acuerdo general sobre los siguientes puntos básicos: la creatividad tiene su origen en el conflicto, surgiendo de la represión de los deseos libidinosos. Las fuerzas del inconsciente que motivan la "solución creadora» igualan a aquellas que motivan la «solución neurótica». La función psíquica y el efecto de la conducta creadora es la descarga de emociones reprimidas hasta que se alcanza un nivel tolerable de balance psíquico. El pensamiento creador se deriva de la elaboración de fantasías "surgidas libremente» y de ideas relacionadas con el ensueño diurno y el juego infantil; mientras la persona creadora acepta esto libremente, la no creadora lo suprime. Cuando los procesos inconscientes se hacen "ego-sintónicos", es posible conseguir una "perfección especial». Se subraya el papel de la experiencia infantil en la producción creativa, siendo considerada la conducta creadora como «una continuación y sustitución del juego infantil» (Getzels y Jackson, 1962, 91-92).

Un aspecto de la formulación freudiana, sin embargo, parece haber atraído menos la atención general. Es el énfasis en «el preguntarse» y en el «impulso de investigación»-en nuestros términos, el encontrar problemas como una característica del proceso creador-. Significativamente, Freud hace hincapié sobre este aspecto en su Leonardo da Vinci. Señala el «infatigable placer de preguntar» observado en niños pequeños, una curiosidad que desaparece en la mayoría de los adultos. La primera fuente de curiosidad es la «investigación sexual infantil». Si este período concluye por medio de la represión, el impulso investigador puede tomar tres cursos diferentes. Puede compartir el destino de la sexualidad, en cuyo caso la curiosidad queda inhibida y reducida de por vida. O la investigación sexual reprimida puede reemerger desde el inconsciente como un razonamiento compulsivo. $\mathrm{La}$ tercera posibilidad, que, en palabras de Freud, es «la más rara y el tipo más perfecto, escapa a la inhibición del pensamiento y al razonamiento compulsivo... La libido escapa del destino de la represión por ser sublimada desde el comienzo en la curiosidad y por el refuerzo del poderoso impulso de investigación...». La curiosidad se puede expresar libremente en forma del interés intelectual y la producción creadora (Freud, 1947, 46-50).

A pesar de las diferencias obvias, estas distintas concepciones del pensamiento creador se encuentran en un paradigma similar del ser humano: una combinación del modelo homeostático del automantenimiento y de la teoría conductual de la reducción de tensión (o conflicto). De acuerdo con este paradigma, el estado natural y óptimo del organismo es una forma de equilibrio, y el organismo actúa siempre de tal manera que vuelve a este estado de balance. Los problemas producen tensiones que llevan al individuo a una actividad mental, lógica o asociativa, para reducir la tensión y el individuo termina la actividad cuando la tensión desaparece con la solución del problema. La ausencia de cierre en una situación genera tensión que incita a la persona a buscar el cierre; y la persona cesa en su acción cuando el balance estructural se recupera. El conflicto consciente $o$ inconsciente produce tensiones que incitan al individuo a perseguir la resolución, y él, o ella, dejan de buscarlo cuando la tensión se alivia. $\mathrm{La}$ actividad mental, el pensamiento, la imaginación, la conducta creadora siempre incluyen reducción de un impulso, una disminución del conflicto, un decremento de la tensión y la estimulación.

Más recientemente se ha propuesto un paradigma básico diferente. Aunque se reconoce que el pensamiento, la solución 
de problemas, la exploración intelectual o la actividad imaginativa pueden ser medios para la reducción de ciertos impulsos, la actividad mental puede ser un fin en sí misma. El organismo puede buscar incrementar la estimulación tanto como decrecerla. Piaget (1951, 1952), Schachtel (1959), Hunt (1961) y White (1959), entre otros, han recalcado que los niños no aprenden mejor cuando tienen hambre o sed; sólo después de haber satisfecho sus necesidades básicas, manifiestan curiosidad y actividad exploratoria. Como sugeriría el modelo homeostático, un niño permanece tranquilo sean satisfechas sus necesidades o no. Por el contrario, es entonces cuando el juego del niño es más vigoroso. Tan pronto como cumple el primer año de vida, un niño muestra evidencia de una exploración activa e incluso experimentación. La conducta es dirigida, selectiva y persistente; es continua no porque sirva a los impulsos primarios, sino porque satisface una necesidad intrínseca de intervenir en el ambiente.

Por otra parte, una porción considerable de la vida adulta se dedica a actividades que, por no desplegar la imaginación, se puede decir que contribuyen al mantenimiento corporal a través de la satisfacción de los impulsos primarios y la obtención de estados de reposo. Como Schachtel dice:

«El motivo principal en la raíz de la experiencia creadora es la necesidad del hombre de relacionarse con el mundo que le rodea... Esto es evidente en el interés del niño pequeño hacia todos los objetos que le rodean, y su exploración y juego con ellos siempre renovados. Es igualmente evidente en el esfuerzo de toda la vida del artista para asir y reflejar algo que ha concebido en su interacción con el mundo, en el deseo de saber del científico sobre la naturaleza de ios objetos que le conciernen y en el interés por las cosas que le rodean de toda la gente que no ha sucumbido el estancamiento en un mundo cerrado de autocentrismo o sociocentrismo" (Schachtel, 1959, 243).

Esta necesidad de exploración y control no se limita a artistas o científicos, sino que se muestra también en actividades comunes, tales como trabajar en enigmas, leer historias de misterio, viajar a paises extranjeros o fantasear, todo lo cual produce placer no por la reducción de impulsos corporales o disminución de conflictos básicos, sino que son perseguidos más bien porque aumentan el nivel de excitación. Como Berlyne sugiere, aunque en un contexto diferente, "pocos impulsos humanos son más inexorables que el ansia de escapar al tedion. O, enfocando el tema más positivamente, pocos impulsos humanos son tan inexorables como la necesidad de novedad. Como Hebb y otros han mostrado, la gente normal necesita tratar con asuntos problemáticos tanto como necesita comida y abrigo. $\mathrm{Y}$ ambicionará lo problemático cuando haya alcanzado un equilibrio homeostático (ver, por ejemplo, Hebb y Thompson, 1954; Bexton, Heron y Scott, 1954; Csikszentmihalyi, 1975).

Lo fundamental de este paradigma alternativo se puede expresar más brevemente como sigue: además de los impulsos viscerogénicos comunes, como el hambre, la sed y el sexo, que se pueden saciar, parece haber unas necesidades neurogénicas, aún pobremente definidas, que son gratificadas por estimulación -necesidades de excitación, variación sensorial y, quizá, sobre todas ellas el reto de la problemática.

Desde este punto de vista, los procesos mentales de los seres humanos no son como mecanismos termostáticos o de procesamiento de información que permanecen ociosos hasta que son disparados por algún estímulo, impulso, tensión o problema presentado; más bien, los procesos mentales humanos son entidades autónomas que deben permanecer activas para funcionar en un nivel óptimo. Los seres humanos no necesitan ser impulsados por presiones externas a explorar, a pensar, a soñar, a imaginar, a perseguir problemas que solucionar; sus funciones mentales ya están constituidas para hacer eso. No son únicamente organismos reductores de estímulos y solucionadores de pro- 
blemas, sino también buscadores de estimulos y descubridores de problemas (Getzels, 1964).

Los dos paradigmas explicativos -la reducción de tensión y la búsqueda de tensión - referidos a la conducta, son maneras diferentes de explicar la motivación para la creatividad. Históricamente, el modelo homeostático surgió como la «tesis» básica de la psicología moderna; el modelo de búsqueda del estímulo se desarrolló a esto como «antítesis». $\mathrm{Ha}$ sido difícil reconciliar las dos; a menudo, una visión prevalece sobre la otra, como cuando la conducta exploratoria se explica como reducción de un «impulso exploratorio», que equivale a una reinterpretación de la búsqueda de estímulo en términos de homeostasis o reducción del impulso. Ya que hay pruebas a favor de uno y otro modelo, ni uno ni otro pueden ser descartados por los fundamentos empíricos. Hasta que surja una síntesis de las dos perspectivas, se puede optar por uno o por otro para considerar las raíces psicológicas de la creatividad.

La pregunta con la que nos enfrentamos es: ¿cuál de las dos explicaciones es más compatible con lo que hemos observado en nuestro estudio sobre los artistas?, ¿está su trabajo motivado por la necesidad de reducir algún impulso instintivo - -sea libidinoso, viscerogénico o exploratorio- o es estimulado por un fin positivo de desafíos crecientes para conseguir niveles más intensos de interacción con el medio ambiente? Al confrontarse con esta elección categórica, uno inmediatamente comprende que ninguna de las dos perspectivas por separado hace justicia a la observación. Ninguna de las dos explica completamente la fuente del descubrimiento de problemas artísticos o la motivación para tratar de resolverlos. $\mathrm{La}$ actividad creadora de los artistas no puede ser explicada adecuadamente por ninguno de los dos modelos. Se necesita una integración de los dos pincipios, una síntesis de posiciones aparentemente antitéticas.

A primera vista puede parecer que el modelo de reducción de tensión es ade- cuado para explicar la conducta creadora de los artistas que hemos estudiado. En su caso, la solución del problema tiende a originar algún conflicto o tensión. La tensión, normalmente, no es percibida conscientemente por el propio artista, aunque puede ser inferida con gran seguridad de la consistencia de su conducta. Por ejemplo, algunos sujetos parecían claramente motivados por el esfuerzo creador como un intento de recuperar la feliz experiencia de la niñez; otros querían resolver dilemas del amor, del tiempo, de la muerte o de la frustración de la vida urbana. No es difícil interpretar su trabajo como una respuesta a las tensiones, como una tentativa de reducir el conflicto o como un impulso para volver a una condición homeostática plácida.

Pero tan pronto como se admite todo esto, también se hace obvia la inadecuación de esta explicación aparente. La fuente de las tensiones que informan del esfuerzo del artista no es tan clara como parece. El conflicto no se debe a un cambio «interno» como cambios fisiológicos en el nivel de glucosa en sangre, o una producción hormonal elevada o la necesidad del sistema nervioso de ser estimulado; tampoco puede explicarse por referencia a la transformación de la energía libidinosa por sí sola o incluso por regresión al servicio del ego (Kris, 1952). Las tensiones que dirigen el trabajo del artista surgen de las relaciones mediadas simbólicamente con el ambiente, con otra gente, con ideas abstractas como el «amor", los «celos", el «orden universal». Si hay un nivel homeostático que restaurar no es sólo un estado de «impulso interno", sino un estado afectivo social y de equilibrio cognitivo con el propio ambiente.

El modelo homeostático supone que las fuentes de tensión (hambre, deseo sexual, necesidad de novedad, etc.) tienen localizaciones concretas en el individuo; están abi prefijadas ya como problemas dentro de los individuos. Pero nuestras observaciones sugieren que esto no es así. El conflicto que dirige al artista existe ini- 
cialmente sólo como una tensión difusa, flotante, sin estructura u objetivo. El proceso creador consiste exactamente en tratar de formular los parámetros del conflicto vagamente percibido en un problema que pueda ser tratado y que pueda resolverse a través de significados simbólicos.

El trabajo de algunos artistas ilustra este proceso claramente. Un artista, por ejemplo, estaba tratando de resolver sus relaciones ambigüas con las mujeres en el momento del estudio. En particular, estaba impresionado por una mujer joven que detrás de una fachada serena parecía tener un oculto temperamente trágico, casi esquizofrénico. En la formulación del problema para el experimento, este artista se veía atraido por los elementos sexualmente sugestivos de los objetos y estructuró con ellos una representación dinámica del conflicto que estaba experimentando. Para formular el problema de esta forma concreta hizo accesibles sus fuentes a la conciencia y, por tanto, responsables de la solución expresiva.

En otro caso, uno de los artistas estaba construyendo una serie de hojas afiladas que, impulsadas por baterias, podían moverse alrededor de la habitación. La entrevista sugirió que la amenaza, el bosque mecánico de cuchillas, estaba relacionado con el subconsciente del artista, con la relación con su padre, cuya influencia veía como una amenaza a su desarrollo personal. El movimiento de las esculturas sirvió como expresión concreta de un conflicto existencial, una expresión que por los significados simbólicos de jó traslucir los sentimientos asociados con el conflicto.

Si el paradigma de la reducción de tensión por sí solo parece inadecuado para explicar la creación artística, excepto en los términos más generales y amorfos (¿Qué conducta no puede ser atribuida a la reducción de tensión?) entonces el modelo de búsqueda del estímulo es por sí solo también inadecuado. Los artistas no están motivados solamente por una necesidad genérica de incrementar estimulación, tampoco por buscar la novedad por sí misma. Se comprometen en actividades exploratorias y, al final, el resultado de su trabajo es algo nuevo, pero la conducta exploratoria es dirigida por el conflicto. Por otra parte, la fuente de la exploración creadora es un conflicto que necesita resolución; esto parece estar en concordancia con el modelo de reducción del estímulo, en el cual la meta de la conducta se entiende como la obtención de una condición de éxtasis anterior al conflicto. Por otro lado, no se trata de una vuelta a una condición inicial que gobierna la acción de la persona creadora, sino de la búsqueda de una nueva condición que está por descubrir - una búsqueda que implica la formulación y descubrimiento de problemas; en este sentido, la conducta del artista encaja con el modelo de búsqueda del estímulo, en el cual el fin de la conducta es lograr una condición emergente.

Si analizamos las pruebas correctamente, la conducta creadora de los artistas sugiere una modificación y síntesis de estos modelos. El arte (o la ciencia, la literatura, la religión y otras formas de actividad simbólica) tienen su fuente en las tensiones. Pero las tensiones, subyacentes al trabajo creador, no son aún problemas estructurados. Para obtener significado y entidad las tensiones inicialmente ambíguas deben ser internalizadas y formuladas como problemas. La tarea crucial de la persona creadora es precisamente la transformación de lo potencial en un problema verdadero. El trabajo creador es la aseveración concreta de problemas existenciales que anteriormente fueron experienciados sólo como tensiones difusas.

Las tensiones que dan origen a la actividad creadora no son ni viscerogénicas, ni estrictamente hablando, psicogénicas; el trabajo no está directamente dirigido a la reducción del desequilibrio psicológico o el alivio de los conflictos libidinosos en la organización de la psique. Uno podría quizá llamarlo sociogénicos, ya que tienen su origen en las ambigüedades simbólicas del tiempo y espacio del artista. En ningún caso, las fuerzas específicas subyacentes a 
la creatividad artística son todavía problemas estructurados, sino que provienen y son partes integrantes del acto mismo de descubrimiento del problema. La dirección de la actividad creadora no se dirige a restaurar un equilibrio previo, sino a alcanzar uno emergente.

Habría que considerar a este respecto el caso del artista cuyos cuadros favoritos tenían que ver con una relación triangular entre él, su madre y esposa. Seguramente, estaba tratando de resolver una tensión emocional. Se podría inferir que estaba intentando recapturar el sentimiento de seguridad que había experimentado en su niñez. Aun así, la figura de la esposa representa un elemento que no había estado alli en su niñez; su presencia en el cuadro demuestra que el artista no solamente retornaba al pasado, sino que despreciaba la realidad presente. Tuvo que encontrar una manera de relacionar los tres elementos conflictivos -o personas- para lograr un equilibrio que nunca había existido antes. Los cuadros son expresiones de su conflicto existencial.

Para otro artista, la fuente principal de tensión parecía mantenerse por los cambios bruscos ocasionados por la guerra. Aquí, una vez más, se han perdido una niñez idílica y un amor materno y su pérdida ha sido dolorosa. Pero, en este caso, el artista también se expuso a un cambio en los valores culturales; después de emigrar a EE.UU., se enfrentó con un estilo de vida extraño. Las cuestiones que ha de resolver se centran en conseguir un nuevo estado de seguridad y armonía, que no niegue ni la realidad presente ni la pasada. El artista no sabe exactamente lo que le "preocupa» ni qué recuerdos extraídos del pasado le pueden ser útiles en el contexto presente. De aquí que construya los problemas en un marco temporal, combinaciones visuales en las que el interés por el pasado y el presente, la realidad y la fantasía interactúan como disfraces simbólicos.

A través del "ensayo-error", el análisis «lógico» y la «inspiración» preconsciente, el artista se encuentra cada vez más cerca del establecimiento del problema específico que su experiencia excepcional ha producido. Puede reconocer o no las fuerzas subyacentes implicadas en el problema que ha creado; es más, algunos artistas dicen que habían comprendido su trabajo sólo después que vieron la «solución» en el lienzo. Probablemente el artista que comprenda a un nivel racional el problema planteado no usará su talento artístico como una forma de tratar con el tema. Cuando el conflicto o la perplejidad son conocidos por la conciencia analítica, cesan de suministrar estímulos para el descubrimiento del problema imaginativo preconsciente. En este caso, la actividad de nuestros artistas se adecúa simultáneamente a los modelos de reducción del estímulo y búsqueda del estímulo; ambos reducirán las tensiones ambigüas a través del descubrimiento de problemas estructurados responsables de las soluciones simbólicas.

La integración de los dos modelos de la actividad creadora se basa en diversos postulados que pueden ahora enunciarse más directamente. La conducta creadora es producida por tensiones generadas dentro o entre símbolos afectivos o cognitivos. Los estados de impulso intrapsíquico no explican suficientemente los productos creativos. Los símbolos que usamos para codificar la experiencia son fuentes de producción imaginativa en sí mismos. Esto sólo no es, por supuesto, un descubrimiento radical: las teorías psicológicas de la disonancia cognitiva, del desarrollo cognitivo y del desarrollo del ego han sugerido antes todo esto. Lo que nuestras observaciones han introducido $y$ subrayado, sin embargo, es la diversidad y complejidad de las experiencias sociales, emocionales e intelectuales productoras de tensiones existenciales que los artistas traducen en problemas creativos palpables.

El sentimiento de aislamiento social del artista inmerso en la situación experimental podría compararse a las propiedades reflectantes de un trozo de cristal fundido. Como los colores de los objetos circundantes se filtran a través del cristal, se 
van a expresar facetas de las relaciones del artista con las personas que conoce. A nivel superficial el problema recuerda el modo como la luz, el color y la forma se ven afectados cuando se mira a través de una pieza irregular de cristal. Pero a un nivel más profundo, el problema devuelve la pregunta existencial que turba a esta persona: ¿Es el mundo como realmente lo ve, o es su percepción la que distorsiona todo al igual que el cristal distorsiona lo que le rodea? Cuando los colores y formas extrañas del desierto encuentran su camino en los cuadros de otro artista, ya no le van a permitir transformar sus tensiones en problemas que pueda tratar artísticamente y deberá buscar medios más afines. Esta visión repentina sugiere la complejidad de la relación entre los conflictos subyacentes y su traducción a obras de arte. Tal relación no se puede explicar por medio de los modelos viscerogénicos o neurogénicos exclusivamente. También debe tenerse en cuenta el poder autónomo de la imaginación humana para crear problemas simbólicos a partir de emociones sin forma.

Los modelos de reducción del estímulo y búsqueda del estímulo presuponen que el origen de un acto sólo puede ser uno entre un número finito de necesidades. Las teorías motivacionales dan por hecho que en la raíz de toda actividad debe haber una causa reducible a una «necesidad" o a un simple conflicto. Estas concepciones no admiten la posibilidad de que puedan surgir a lo largo de la vida de una persona o en el curso de la historia nuevas exigencias existenciales, irreductibles a «causas» previas. La integración propuesta no niega la significación de los impulsos viscerogénicos universales y las necesidades psicológicas. Gran parte de nuestra conducta se relaciona con el mantenimiento y la restauración del equilibrio fisiológico y emocional.

De forma similar han evolucionado ciertas instituciones muy difundidas, como la familia nuclear, que produce tensiones básicas, como el complejo de Edipo. Pero, estas "causas" pueden tan sólo dar explicaciones aproximadas y muy genéricas. Hay que tener en cuenta, también, las tensiones afectivas y las experiencias cognitivas que pueden aparecer en la vida del individuo que existe en un momento histórico concreto: las tensiones y experiencias producidas por la vida urbana, las situaciones políticas inauditas, las nuevas relaciones familiares, los descubrimientos científicos y los cambios de estilos estéticos.

En el modelo homeostático y en la teoría de la descarga del impulso, el acto creador simplemente restaura el estado psicológico que existía antes del acto. Desde este punto de vista, el logro de Picasso no es cualitativamente diferente del de Praxíteles, el trabajo de Einstein es psicológicamente equivalente al de Demócrito. $\mathrm{La}$ creatividad deviene un término inapropiado, ya que el supuestamente acto creador no altera nada; simplemente restaura un estado natural que no es afectado por el paso del tiempo, el cambio cultural o los nuevos avances. Toda la profundidad histórica se pierde: cada realización o logro humano se reduce a la misma matriz de necesidades básicas. Una vez más, no contradecimos que haya necesidades de equilibrio básicas que dirigían a la gente a la acción en la antigua Grecia como hoy en día, pero también parece obvio que además hay necesidades emergentes hacia realizaciones que construyen, incorporan y nos llevan más allá del viejo equilibrio.

La creatividad implica sensibilidad a tensiones no formuladas previamente $y$ habilidad para expresarlas como problemas que se pueden solucionar. Pero, explicar este proceso en términos de un modelo cerrado de necesidades distorsiona la realidad que se va a explicar. Tampoco tiene sentido entender el proceso en términos de una tendencia generalizada de explorar, producir novedad o buscar estímulos. Ambas concepciones son necesarias cuando se integran en un modelo dialéctico. Podríamos, por tanto, describir la creatividad artística como sigue: es el proceso por el que un individuo, 1) experimenta un conflicto en la percepción, emoción o pensamiento, 2) 
formula un problema articulando el conflicto previamente inarticulado, 3) expresa el problema de una forma visual, 4) triunfa en la resolución del conflicto a través de significados simbólicos y 5) en consecuencia, logra un nuevo equilibrio cognitivo y emocional. La meta del acto creador no es reducir un impulso para restaurar un equilibrio previo, sino conseguir un nuevo equilibrio. La clave del logro creador es la transformación de un conflicto intangible en un problema simbólico palpable para el cual la solución creadora será la respuesta.

Estas son las cuestiones aportadas por nuestro estudio. Para tratarlas de forma concluyente necesitaríamos conocer mucho más sobre las clases de tensiones que perturban los procesos homeostáticos -tanto los emergentes como aquellos que son constantes-. Necesitamos saber mucho más sobre cómo las tensiones vagas se transforman en problemas articulados y cómo se expresan simbólicamente. Mientras tanto, la observación del artista en su trabajo nos provee de algunas ideas acerca de lo que está implicado en el proceso de descubrimiento de problemas. En conjunto, estas observaciones nos sugieren un proceso que, hasta ahora, no ha sido estudiado sistemáticamente.

El proceso creador es estimulado por tensiones que no se pueden expresar en los términos simbólicos o conceptuales que ya existen. Si pudieran expresarse en términos ya conocidos, el resultado del problema no sería original. Incluso, en los límites de la situación experimental, los artistas que empezaron a concebir su dibujo con una formulación personal (por ejemplo, el uso de una pieza de cristal para representar el filtro de las relaciones interpersonales) producían más trabajo original que aquellos que empezaban con una formulación conocida (tal y como la representación de «un punto inicial que se desvanece»). Consciente o inconscientemente, el artista creador transforma sus tensiones emocionales profundamente sentidas en una contrapartida expresiva, tangible; cuando ha ordenado estos ele- mentos simbólicos en una relación dinámica apropiada, ha encontrado el problema creador.

Parece ser esencial para el artista considerar diversos elementos expresivos potenciales, aunque más tarde sólo utilice unos pocos. Si se restringe a unos pocos elementos desde el principio, el problema que estructura va a ser probablemente menos original. El artista debe ser sensible a muchas formas visuales; si sólo responde a objetos entendidos convencionalmente como bellos, o sólo a sus características estéticas obvias, probablemente va a formular un problema "presentado» -es decir, aquel que se define por reglas conocidas- más que un problema «descubierto", que incluye relaciones nuevas entre los elementos en conflicto.

Los artistas que definian su problema poco después de empezar a trabajar producían dibujos que eran menos originales que aquellos que mantenían el problema abierto más tiempo. Parece que el problema creador no puede ser totalmente visualizado en "el ojo de la mente»; debe ser descubierto por la interacción con los elementos que lo constituyen. Aplazar la solución ayuda a garantizar que el artista no se quedará en un problema superficial o gastado. Ahora bien, este aplazamiento no se debe a la falta de dirección; nuestras observaciones nos sugieren que aquellos que esperan para estructurar su pintura, trabajan por una meta definida, aunque ésta no sea accesible al conocimiento consciente. El éxito artístico en nuestro grupo estuvo más fuertemente relacionado con esta fase del proceso de encontrar problemas, que probablemente se convierta en uno de los temas centrales de la creatividad en general.

Además, para evitar una cristalización de la estructura del problema demasiado temprana, la mayoría de los artistas creadores, experimentaban con varias estrategias de solución de problemas en el transcurso de la tarea. Se puede decir que la solución del problema artístico consiste en la combinación armoniosa o consistente de varios elementos simbólicos, ex- 
presivos y formales en et lienzo. Al mismo tiempo, como señaló Wertheimer, en un trabajo creador la formulación del problema sólo llega a manifestarse en la solución del mismo. La formulación y la solución se darán al mismo tiempo. En nuestro experimento, el artista que trataba diferentes medios de solución -experimentaba con diferentes pigmentos, arreglos, superficies, herramientas- tenía más probabilidades de descubrir una nueva manera de representar su problema, produciendo una solución más original.

Finalmente, otra concomitancia del proceso creador en el arte es la actitud del artista hacia la «solución». Los artistas creativos no sólo desconocían los parámetros exactos del problema hasta que el dibujo estaba casi terminado, sino que estaban también inseguros mientras se terminaba y cuando se terminó. Esto es, de nuevo, consistente con la naturaleza de los problemas creadores. Las tensiones involucradas en los conflictos existenciales básicos nunca se pueden expresar de una vez por todas. Cada formulación sólo puede ser parcial, debe tratar solamente con unas pocas dimensiones de la experiencia, solamente con una selección de los posibles puntos de vista. Una persona que asegura que su pintura ha terminado - el problema solucionado totalmente de modo que no se puede alterar- debe creer que ha encontrado la solución correcta; pero la solución correcta sólo existe para problemas que han sido solucionados antes.

En principio, el mismo paradigma de encontrar problemas parece poder aplicarse a otras formas de creatividad distintas de la artística. Muchos científicos destacados dicen que la formulación de un problema es más esencial que su solución y que es un acto más imaginativo. El contenido específico del proceso de encontrar problemas variará dependiendo de la forma que las tensiones, conflictos y dilemas tomen -ya sea perceptual, teórica, social, política, mecánica o cualquier otra-. Pero, sin tener en cuenta de qué campo de problemas se trata, si una per- sona aproxima su tarea a un camino creador o no depende de los siguientes criterios: ¿Se aproxima a la tarea como una situación problema presentada, en la que los elementos problemáticos (los que producen la tensión) ya se conocen, o como una situación problema descubierta, en la que el elemento crucial no se conoce todavía o se excluye?, ¿define el problema, es decir, lo que debe ser hecho de una manera rutinaria, o siente que puede haber cosas por hacer y que no están claras todavía?, ¿utiliza sólo unos pocos elementos familiares simbólicos para construir la definición del problema, o considera diversas alternativas?, en el trabajo hacia la solución, ¿parece ya conocer lo que debe ser el resultado o se mantiene redefiniendo el problema por medio de la interacción con los elementos simbólicos?, ¿se fía de una estrategia sencilla, familiar, de solución de problemas, o atiende a diversas estrategias, esto es: acepta un método cerrado desde el principio o puede llegar a aplazar el cierre metodológico?, cuando el problema se resuelve aparentemente: ¿se contenta con la solución o piensa que ésta es tan sólo un paso hacia otros enunciados quizá más fructíferos de un problema todavía por resolver?

Si se sustituyen términos concretos por variables abstractas como «lo que se debe hacer», «elementos problemáticos» y «alternativas", que son obviamente diferentes para los distintos campos, entonces las cuestiones de esta clase proporcionan una guía para observar el nivel de creatividad con que una persona se aproxima a una tarea dada. La lista no es exhaustiva, por supuesto, incluye únicamente las variables que, dentro de los límites de nuestro diseño experimental, parecen estar referidas al éxito creador del artista.

Supongamos que se deseara aplicar este modelo a los científicos o, más específicamente, a los matemáticos. ¿Cuáles son los elementos problemáticos para ellos, números, fórmulas, derivadas, algunas relaciones extrañas entre fuerzas y cantidades? y, ¿cómo podría saber un observador si un matemático explora un número ma- 
yor de elementos relevantes que otro? En general, parecería más difícil desarrollar un test abstracto de encontrar problemas, que uno de inteligencia o solución de problemas. Encontrar problemas parece depender de la sensibilidad y el conocimiento profundo de un campo específico de experiencia; por ello, es dudoso que sea factible un test de aptitud general para encontrar problemas. Pero esto no quiere decir que el descubrimiento de problemas y su relación con la originalidad de las soluciones no pueda investigarse en otros campos sobre las líneas de nuestro estudio de artistas jóvenes.

El mismo modelo y el mismo método han empezado, de hecho, a ampliarse al descubrimiento de problemas en otros campos. Por ejemplo, en un estudio reciente se enfrentó a estudiantes con un problema del tipo Duncker de ordenar objetos (como en el estudio de los artistas) y se les pidió inventar cuestiones y formular problemas con este material. $\mathrm{La}$ cantidad y calidad de los problemas propuestos estaban relacionadas con características personales e intelectuales de los sujetos. En la muestra de 60 sujetos, el número de problemas planteados por cada persona varió de 2 a 31. Cuando la calidad de los problemas se clasificaba en una escala de seis puntos de «simple» a "complejo", el rasgo varió desde una media individual de 1,4 a 4,7. Se encontraron relaciones significativas entre la calidad de los problemas y la calidad del funcionamiento cognitivo reflejado en medidas de pensamiento y formación de conceptos. Asi pues, hay diferencias entre los individuos en la aptitud para encontrar problemas (como las hay en la aptitud para resolverlos) y la aptitud de encontrar problemas se relaciona con las características personales del sujeto (Arlin, 1974).

Se han comenzado a hacer estudios similares en el campo de la interacción social. En un estudio se les pidió a los sujetos que formularan problemas interpersonales y se encontraron correlaciones entre la cantidad y calidad de los problemas y las medidas cognitivas y de personalidad. En un segundo estudio, la manera en que los problemas se planteaban se relacionaba con la forma en que los mismos eran resueltos. Comentar los resultados de estos estudios es prematuro, toda vez que aún están en marcha. Pero, prescinciendo de que la situación problemática se refiera a objetos o a personas, parece que hay diferencias individuales en el estilo en que los problemas se encuentran y se formulan, así como en la cantidad y calidad de los problemas planteados. Hay también relaciones sistemáticas en el estilo, cantidad y cualidad de los problemas que una persona encuentra y sus rasgos cognitivos y de personalidad.

Estas ilustraciones esbozadas, junto con el estudio con artistas, demuestran que debe estudiarse empíricamente el proceso de encontrar problemas y su papel crucial en el pensamiento y la creatividad. Encontrar problemas puede ser tan importante como la solución de problemas para entender la creatividad; el don del genio no es solamente la posesión de habilidades técnicas o la facilidad para resolver problemas, sino también la sensibilidad e imaginación para encontrarlos.

Bien podemos preguntarnos: «inecesitan los problemas ser encontrados?, ¿no hay ya suficientes conflictos y dilemas inter e intrapersonales en casa, en los negocios, en las artes y en las ciencias? Por supuesto que hay un exceso de intranquilidad, mucha ansiedad causada por conflictos propios de nuestro tiempo. Pero muchos de ellos no se nos presentan como problemas que puedan ser resueltos - ni siquiera contemplados de forma aprovechable. La intranquilidad se debe solucionar por caminos productivos para alcanzar una solución.

Los máximos resolutores de problemas de hoy en día son las máquinas que procesan información (Mackworth, 1965; Getzels y Dillon, 1973). Pero la cantidad y la calidad de los descubrimientos dependen de la gente que puede articular tensiones vagas, convirtiéndolas en problemas significativos. Algunos individuos (como el imitador en el arte, el técnico en la ciencia, el alumno pedante, el gobernador bu- 
rócrata) se enfrentan a problemas que ya han sido identificados. El buen artista, el científico inventor, el escolar creativo, el hombre de estado innovador, la persona autorrealizada son los que son conscientes de los problemas no formulados, que están potencialmente presentes en los conflictos de su propia experiencia: a diferencia de las máquinas, idean sus propios programas y trabajan sobre problemas descubiertos.

Esta habilidad no está basada en una superioridad cuantitativa de la memoria, el razonamiento o las capacidades cognitivas convencionales. La habilidad de formular problemas parece ser una facultad de orden diferente. Supone un proceso mucho más relacionado con las capas profundas del ser y no sólo con la razón; es mucho más holístico, ya que implica al estado experimental total de la persona. El proceso está dirigido al objetivo, pero a veces persigue metas por debajo del umbral de la conciencia. Busca similitudes entre los objetos externos y los estados internos; usa significados simbólicos para expresar sentimientos sin forma respecto de ese descubrimiento que de otra manera sería imperceptible, articulando lo que de otro modo quedaría desarticulado. Bien podría estar el proceso de encontrar problemas en el origen de la visión creadora. A pesar de su vaguedad, este proceso merece que se le dedique la misma atención otorgada a otros componentes del pensamiento más conocidos y accesibles.

\section{Referencias}

ARLIN, P. K.: Problem finding: The relation between cognitive variables and problem fingind performance. Tesis sin publicar. Universidad de Chicago, 1974.

BERLYNE, D. E.: «Novelty and curiosity as determinants of exploratory behavior». British Journal of Psycho$\log y, 1950-1951,41,68-80$.

BEXTON, W. H.; HeRON, W. G., y SCOTT, J. H.: «Effect of decreased variation in sensory environment». Canadian Journal of Psychology, 1954, 8, 70-76.

Csiksz.ENTMIHALY, M.: Beyond Boredom and Anxiety: The Experience of Play in Work and Games. Jossey-Bass. San Francisco, 1975.

Freud, S.: Leonardo da Vinci: A Study in Psychosexuality. Nueva York: Random House, 1947.

GET/ELS, J. W.: «Creative thinking, problem-solving, and instruction". En E. R. Hilgard (ed.): Theories of Learning and Instruction, 63rd Yearbook of the National Society for the Study of Education, Part 1. Chicago: Chicago Press, 1964, 240-267.

GetLels, J. W., y Dillon, J. T.: «The nature of giftedness and the education of the gifted». En R. M. W. Travers (ed.): Second Handbook of Research on Teacbing. Chicago: Rand McNally, 1973, 689-731.

GeTZELS, J. W., y JACKSON, P. W.: Creativity and Intelligence: Explorations with Gifted Students. Nueva York: Wiley, 1962.

HUNT, J. MCV.: Intelligence and Experience. Nueva York: Ronald, 1961.

KRIS, E.: Psychoanalytic Explorations in Art. Nueva York: International University Press, 1952.

MaCkworth, N. H.: "Originality". American Psycbologist, 1965, 20, 51-56.

PiaGeT, J.: The Origins of Intelligence in Children. Nueva York: International Universicy Press, 1952 (original, 1936).

PIAGET, J. PlaY: Dreams, and Imitation in Childbood. Nueva York: Norton, 1951 (originad, 1945).

SCHACHTEl, E. G.: Metamorphosis: On the Detelopment of Affect, Perception Attention and Memory. Nueva York: Basic Books, 1959.

THORNDIKE, E. L.: Measurement of Intelligence. Nueva York: Bureau of Publications, Teacher College, Columbia University, 1962.

WERTHEIMER, M:: Productive Thinking. Nueva York: Harper \& Row, 1945.

WHITE, R. W.: "Motivation reconsidered: The concept of competence». Psychological Review, 1959, 66, $297-331$. 